\title{
Drug delivery in biological tissues: a two-layer reaction-diffusion-convection model
}

Sean McGinty and Giuseppe Pontrelli

\begin{abstract}
In this paper we present a general model of drug release from a drug delivery device and the subsequent drug transport in biological tissue. In order to study the complete process, a two-phase mathematical model describing the transport of a drug between two coupled media of different properties and dimensions is presented. A system of partial differential equations describes both the solid-liquid transfer and diffusion processes in the polymeric substrate as well as diffusion, convection and reaction in the tissue layer. The model serves as a useful tool for providing insight into the concurrent effects of the diffusion, convection, solid-liquid transfer and reaction parameters in drug delivery systems.
\end{abstract}

\section{Introduction}

Local drug delivery devices (DDD) have received much attention in recent years, since they provide a convenient means of targeting drug at the site where it is needed most. Historically, drugs have been administered either orally, topically or hypodermically, and often by the patients themselves. The advent of local DDD has meant that drug delivery can be more controlled, with a prescribed amount of drug being delivered over the necessary time period, and with less input required from the patient. Whilst the drug delivery may in principle be monitored, it is often unclear how the DDD can be designed to achieve the level of control required for a specific purpose, since there exists complex interplay between biology, polymer chemistry and pharmacology [1].

Important examples of local DDD include drug-eluting stents for the prevention of restenosis following percutaneous coronary intervention [2], therapeutic contact

Sean McGinty

Strathclyde University, Glasgow, UK, e-mail: s.mcginty@strath.ac.uk

Giuseppe Pontrelli (corresponding author)

IAC-CNR, Rome, Italy, e-mail: giuseppe.pontrelli@gmail.com 

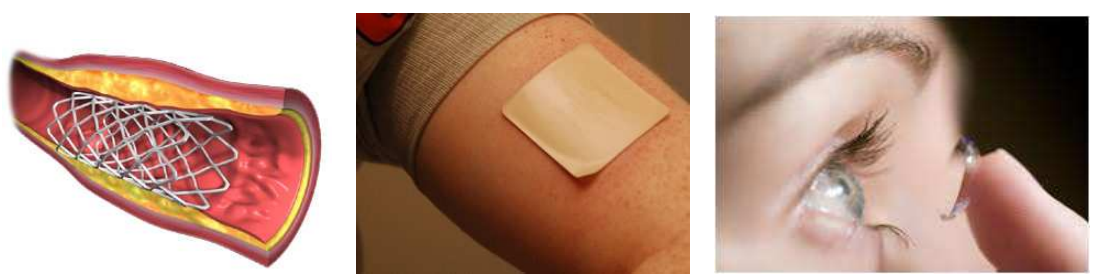

Fig. 1 Three examples of DDD: the drug-eluting stent (left), the transdermal patch (center), the therapeutic contact lens (right).

lenses to deliver ophthalmic drugs [3], and transdermal drug delivery [4] (Fig. 1). In each case, the drug is commonly contained in a polymeric gel platform that is in direct contact with the biological tissue. The polymeric gel acts as a reservoir of drug and provides an adjustable level of control over the rate of drug delivery to the tissue [5]. Both the polymeric gel layer and the interfaced tissue are treated as porous media from a macroscopic point of view. The therapeutic success is dependent on the extent of drug elution, the rate of release, accumulation of drug and binding to components within the tissue [6]. Furthermore, the local drug concentrations achieved are directly correlated with biological effect and local toxicity. The pharmacological effects of the drug, tissue accumulation, duration and distribution could potentially have an effect on its efficacy and a delicate balance between adequate amount of drug delivered over an extended period of time and minimal local toxicity should be found [7].

Mathematical modelling can serve as an extremely useful tool for providing insight into the important parameters in the system, and to give an indication of how the device may be modified to achieve the desired drug release profile. Many studies on DDD have been conducted regarding efficacy and optimal design either with experimental methods, or modelling/numerical simulations, or a combination of both [8-11]. Nonetheless, many questions remain unanswered for bioengineers and pharmaceutics developers who continue to explore and evaluate this technology. In particular, finding the optimum dose to be delivered in a personalized way to a specific tissue still remains a significant challenge.

In this paper we present a general mathematical model of drug release from a DDD and the subsequent drug transport in a biological tissue. In order to study the complete process, a two-phase mathematical model describing the dynamics of a drug between two coupled media of different properties and dimensions is proposed. A system of partial differential equations describes both the solid-liquid transfer and diffusion processes in the polymer as well as diffusion, convection and reaction in the tissue layer. 


\section{Formulation of the problem}

In a typical DDD, the mass dynamics occurs across a two-layered system composed of: (i) a polymeric platform, acting as a reservoir, where the drug is initially contained, and (ii) the biological tissue (the release medium) where the drug is directed, and exerts a therapeutical effect (Fig. 2).

Layer (i) is shaped as a planar slab, enclosed on one side with an impermeable backing and having the other side in contact with layer (ii). A rate-controlling membrane protecting the polymer matrix may exist at the interface (i)-(ii). It is important to emphasize here that the drug dynamics intrinsically depends on the properties of the coupled system. As most of the mass dynamics occurs along the direction normal to the tissue surface, the modelling may reasonably be restricted to a simplified one-dimensional case. In particular, we consider the $x$-axis to be normal to the layer surface and oriented with the positive direction outwards. Without loss of generality, let $x_{0}=0$ be the interface and let $l_{0}$ and $l_{1}$ be the thicknesses of the layers (i) and (ii) respectively, with $l_{1} \gg l_{0}$ (Fig. 2). The layers are both treated from a macroscopic perspective so that they are represented as two homogeneous porous media.

Preliminary definitions and nomenclature

In the continuum approach, a porous medium can be represented as a homogeneous material by defining averaged variables over a large enough volume, the representative elementary volume (r.e.v.) $V_{\text {rev }}$, of size larger than the pore scale, but smaller than the typical length scale of the phenomenon. Let us define porosity, $\varepsilon$, as the ratio of void volume $\left(V_{\text {rev }}^{f}\right)$ to total volume $\left(V_{\text {rev }}=V_{\text {rev }}^{f}+V_{\text {rev }}^{s}\right)$. It may be that not all void space is accessible to solute. For example, the pore size may be smaller than the size of the solute molecules, or some of the voids may be isolated and not connected to other pores. Thus we introduce a new parameter, the partition coefficient $(k)$, such that $k \varepsilon$ represents the available void volume. We indicate by $\phi$ the ratio of accessible void volume to solid volume:

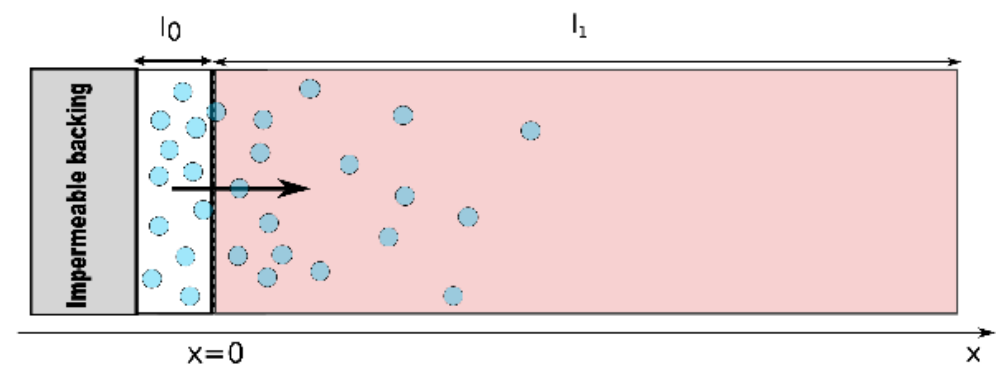

Fig. 2 Cross section of a typical DDD, showing the schematic drug delivery mechanism between polymer and tissue, the geometrical configuration and the reference system. Due to an initial difference of free drug concentrations $c_{0}$ and $c_{1}$, a mass flux is established at the interface and drug diffuses through the biological tissue. Figure not to scale. 


$$
\phi=\frac{k \varepsilon}{1-\varepsilon} .
$$

When defining the concentration, two different ways of averaging over a volume exist. One is based on the volume of each phase contained in r.e.v., that is $k V_{\text {rev }}^{f}$, for the accessible fluid-phase ${ }^{1}$ (which is the fraction $k \varepsilon$ of the r.e.v.) and $V_{\text {rev }}^{s}$ for the solid-phase (which is the fraction $1-\varepsilon$ of the r.e.v.). Another way is to average over the whole $V_{\text {rev }}=V_{\text {rev }}^{f}+V_{\text {rev }}^{s}$ [12]. In the first case, we refer to intrinsic volumeaveraged drug concentration in accessible fluid and solid phases $c^{f}$ and $c^{s}(\mu \mathrm{g} / \mathrm{ml})$, in the second, to volume-averaged drug concentrations, $c$ and $c^{*}$. They are related by the relationships:

$$
c=k \varepsilon c^{f}, \quad c^{*}=(1-\varepsilon) c^{s} .
$$

\section{Modelling drug dynamics in the polymer (delivery)}

Initially, the drug exists wholly in a solid phase encapsulated within the polymer matrix (e.g. nanoparticles or crystalline form $)\left(c_{0}^{s}\right)$ : in such a state, it is unable to be delivered to the tissue. When exposed to biological fluid, as in the in-vivo case, the polymer becomes wetted, initiating a solid-liquid mass transfer (dissolution) process, providing a means for the drug to elute from the device. The rate of transfer of drug from the solid phase to the biologically available free phase $\left(c_{0}^{f}\right)$ is proportional to the difference between $c_{0}^{s}$ and $c_{0}^{f}$. Since the diffusion of drug in the solid phase is negligible, it can be shown that the equations for the drug dynamics in layer (i) in terms of volume averaged concentrations (2) are:

$$
\begin{array}{lrl}
\frac{\partial c_{0}^{*}}{\partial t}=-\alpha_{0}\left(\phi_{0} c_{0}^{*}-c_{0}\right) & \text { in }\left(-l_{0}, 0\right) \\
\frac{\partial c_{0}}{\partial t}=D_{0} \frac{\partial^{2} c_{0}}{\partial x^{2}}+\alpha_{0}\left(\phi_{0} c_{0}^{*}-c_{0}\right) & \text { in }\left(-l_{0}, 0\right)
\end{array}
$$

where $D_{0}\left(\mathrm{~cm}^{2} \cdot \mathrm{s}^{-1}\right)$ is the effective diffusion coefficient of the solute and the solidliquid rate parameter $\alpha_{0}\left(s^{-1}\right)$ incorporates a mass transfer coefficient and the hydraulic radius (free flow area over wetted perimeter) [12].

\section{Modelling drug dynamics in the tissue (absorption)}

Following the solid-liquid transfer process, the now biologically available drug diffuses through the polymeric layer and crosses the interface into the biological tissue.

${ }^{1}$ Superscripts $s$ and $f$ denote solid and fluid phases, respectively. Subscripts 0 and 1 indicate layers (i) and (ii) respectively. 
This is typically comprised of several layers of different size and composition, but for the purposes of this paper we consider tissue as a homogeneous single-layered medium. However, the model may be extended to multi-layers following the approach of [13]. Within the release medium the free drug undergoes diffusion and, in many cases, convection due to a pressure difference across the tissue. In addition, the drug may bind reversibly to specific components of the tissue: indeed, the drug is often targeted to bind to specific receptors on the surface of or within cells. These binding/unbinding processes are often referred to as association/dissociation. We model the binding/unbinding process as a first order reaction, similarly to the liquid/solid mass transfer in (3)-(4). However, in contrast to layer (i), we account for different rates between the forward and reverse reactions, say $\beta_{1}$ and $\delta_{1} \geq 0$ $\left(s^{-1}\right)$ which, here, are defined as the inverse of the forward and reverse reaction time scales, respectively. They are related through the equilibrium dissociation constant, $K_{1}=\frac{\delta_{1}}{\beta_{1}}$, a parameter which can typically be measured experimentally [14]. Here we denote the free and bound drug concentrations as $c_{1}^{f}$ and $c_{1}^{s}$ respectively. Thus, in the biological tissue, the two-phase drug dynamics is governed by the linear reaction-convection-diffusion equations in terms of volume averaged concentrations (2):

$$
\begin{array}{ll}
\frac{\partial c_{1}}{\partial t}=D_{1} \frac{\partial^{2} c_{1}}{\partial x^{2}}-v \frac{\partial c_{1}}{\partial x}-\delta_{1}\left(\frac{c_{1}}{K_{1}}-\phi_{1} c_{1}^{*}\right) & \text { in }\left(0, l_{1}\right) \\
\frac{\partial c_{1}^{*}}{\partial t}=\delta_{1}\left(\frac{c_{1}}{K_{1}}-\phi_{1} c_{1}^{*}\right) & \text { in }\left(0, l_{1}\right)
\end{array}
$$

where $D_{1}$ is the effective diffusivity of unbound drug and $v\left(\mathrm{~cm} \cdot \mathrm{s}^{-1}\right)$ is the magnitude of the convection which is assumed to act in the positive $x$ direction. We have made the assumption that the drug does not diffuse within the components to which it is bound.

We note that the first order linear reaction model of the binding/unbinding process in (5)-(6) may not be the most suitable in all circumstances. In some applications of DDD, such as in drug-eluting stents, a second-order saturable reversible binding model has been proposed to describe the binding of limus compound drugs to arterial tissue [6]: this comprehensive model includes a number of drug dependent parameters which are difficult to measure experimentally and, nevertheless, does not necessarily apply in all DDD. Even in cases where a non-linear model is generally more appropriate, the linear model with suitably chosen parameter values can be shown to suffice in certain circumstances (i.e at early times and for sufficiently high initial drug concentrations and binding site density). 


\section{Boundary, interface and initial conditions}

To close the previous two-layer mass transfer system of eqns. (3)-(6), a flux continuity condition has to be assigned at the layer (i)-(ii) interface:

$$
-D_{0} \frac{\partial c_{0}}{\partial x}=-D_{1} \frac{\partial c_{1}}{\partial x}+v c_{1} \quad \text { at } x=0
$$

As far as the concentration continuity is concerned, this is not guaranteed because of a different drug partitioning between the two layers [13]. Additionally, a semipermeable rate-controlling membrane or a non-perfect contact, having $1 / P$ as mass resistance, might be present at the interface. Thus, a jump concentration may occur:

$$
-D_{1} \frac{\partial c_{1}}{\partial x}=P\left(\frac{c_{0}}{k_{0} \varepsilon_{0}}-\frac{c_{1}}{k_{1} \varepsilon_{1}}\right) \quad \text { at } x=0,
$$

with $P\left(\mathrm{~cm} \cdot \mathrm{s}^{-1}\right)$ the overall mass transfer coefficient [13].

No mass flux passes to the outer surrounding due to the impermeable backing and we impose a no-flux condition :

$$
D_{0} \frac{\partial c_{0}}{\partial x}=0 \quad \text { at } x=-l_{0}
$$

Finally, a boundary condition has to be imposed at the external boundary. Depending on the thickness of the tissue, the concentration may vanish at this point. However, we propose the more general Robin boundary condition (from which $c_{1}=0$ can be recovered by choosing $\gamma$ sufficiently large):

$$
-D_{1} \frac{\partial c_{1}}{\partial x}+v c_{1}=\gamma c_{1} \quad \text { at } x=l_{1}
$$

The initial conditions are:

$$
c_{0}^{*}(x, 0)=C_{e} \quad c_{0}(x, 0)=0 \quad c_{1}(x, 0)=0 \quad c_{1}^{*}(x, 0)=0 .
$$

\section{Model solution}

The model we have presented is very general and may be applied to several DDD. It is often useful to write a model in non-dimensional form. A typical nondimensionalization for a system of reaction-diffusion-convection equations leads to three important numbers: the Péclet number, the first Damköhler number and the second Damköhler number. These dimensionless groups define, respectively, the relative importance of convection to diffusion, of reaction to convection, and of reaction to diffusion. By examining their size, it is often possible to simplify the model by neglecting parameters that are unimportant. For example, in the case of 
drug-eluting stents, while there exists a small convective flow due to the transmural pressure gradient across the arterial wall, the Péclet number is often small, meaning that the convective term can be reasonably neglected. Depending on the particular DDD, it may also be possible to neglect the solid-liquid mass transfer terms if the timescale for this process is far shorter than that of diffusion in the polymer. Similarly, if the timescale for reaction is far shorter than that of diffusion and convection, then the reaction may be considered instantaneous, in which case the two phases in the tissue can be assumed to be in dynamic equilibrium. When the model has been simplified as far as can be, an analytical or semi-analytical solution may be obtained by using the techniques described in $[13,15,16]$, or alternatively, an appropriate numerical procedure can be used.

\section{Summary}

In this paper we have presented a general model of drug release from a DDD and the subsequent drug transport in biological tissue. The model is based on a twolayer two-phase system where a system of partial differential equations describes both the solid-liquid transfer and diffusion processes in the polymeric layer as well as diffusion, convection and reaction in the tissue layer. We have indicated how the model may be tuned and simplified to suit the required application and have suggested suitable solution techniques.

\section{References}

1. Siepmann, J. Siepmann, F.: Mathematical modeling of drug delivery, Int. J. Pharm., vol. 364 (2), pp. 328-343 (2008).

2. McGinty, S., McKee, S., Wadsworth, R.M., McCormick, C.: Modelling drug-eluting stents, Math. Med. Biol., vol. 28, pp. 1-29 (2011).

3. Zhang, W.E., Prausnitz, M.R., Edwards, A.U.: Model of transient drug diffusion across cornea, J. Control. Release, vol. 99, pp. 241-258 (2004).

4. Prausnitz, M.R., Langer, R.: Transdermal drug delivery, Nat. Biotechnol., vol. 26(11), pp. 12611268 (2008).

5. Langer, R.: Polymeric delivery systems for controlled drug release, Chem. Eng. Commun., vol. 6, pp. 1-48 (1980).

6. Tzafriri A.R., Groothuis A., Price G.S., Edelman E.R.: Stent elution rate determines drug deposition and receptor-mediated effects, J. Control. Release, vol. 161, pp. 918-926 (2012).

7. Creel, C.J., Lovich, M.A., Edelman, E.R.: Arterial paclitaxel distribution and deposition, Circ. Res., vol. 86 (8), 879-884 (2000).

8. Siepmann, J., Göpferich, A.: Mathematical modeling of bioerodible, polymeric drug delivery systems, Adv. Drug Delivery Rev., vol. 48, pp. 229-247 (2001).

9. Crane, M., Hurley, N.J., Crane, L., Healy, A.M., Corrigan, O.I., Gallagher, K.M., McCarthy, L.G.: Simulation of the USP drug delivery problem using CFD: experimental, numerical and mathematical aspects, Simul. Model. Pract. \& Theory, vol. 12 (2), pp. 147-158 (2004).

10. Narasimhan, B.: Mathematical models describing polymer dissolution: consequences for drug delivery, Adv. Drug Delivery Rev., vol. 48, pp. 195-210 (2001). 
11. Bozsak, F., Chomaz, J.M., Barakat, A.I.: Modeling the transport of drugs eluted from stents: physical phenomena driving drug distribution in the arterial wall, Biomech. Model. Mechanobiol., vol. 13(2), 327-347 (2014).

12. de Monte, F., Pontrelli, G., Becker, S.M.: Drug release in biological tissues, Chapt. 3 in: Becker S.M., Kuznetsov A. V. (Eds), Transport in Biological Media, Elsevier, pp. 59-118 (2013).

13. Pontrelli, G., de Monte, F., A multi-layer porous wall model for coronary drug-eluting stents, Int. J. Heat Mass Transf., vol. 53, pp. 3629-3637 (2010).

14. Anissimov, Y.G., Jepps, O.G., Dancik, Y., Roberts, M.S.: Mathematical and pharmacokinetics modelling of epidermal and dermal transport processes, Adv. Drug Delivery Rev., vol. 65, pp. 169-190 (2013).

15. Pontrelli, G., Di Mascio, A., de Monte, F.: Local mass non-equilibrium dynamics in multilayered porous media: application to the drug-eluting stents, Int. J. Heat Mass Transf., vol. 66, pp. 844-854 (2013).

16. McGinty, S., McKee, S., Wadsworth, R.M., McCormick, C.: Modeling arterial wall drug concentrations following the insertion of a drug-eluting stent, SIAM J. Appl. Math., vol. 73(6), pp. 2004-2028 (2014). 